\title{
The transmembrane domain of HIV-1 Vpu is sufficient to confer anti-tetherin activity to SIVcpz and SIVgor Vpu proteins: cytoplasmic determinants of Vpu function
}

\author{
Silvia F Kluge ${ }^{1}$, Daniel Sauter ${ }^{1}$, Michael Vogl ${ }^{1}$, Martine Peeters ${ }^{2}$, Yingying Li $^{3}$, Frederic Bibollet-Ruche ${ }^{3}$,
} Beatrice $\mathrm{H} \mathrm{Hahn}^{3}$ and Frank Kirchhoff ${ }^{*}$

\begin{abstract}
Background: The acquisition of effective Vpu-mediated anti-tetherin activity to promote virion release following transmission of SIVcpzPtt from central chimpanzees (Pan troglodytes troglodytes) to humans distinguishes pandemic HIV-1 group M strains from non-pandemic group N, O and P viruses and may have been a prerequisite for their global spread. Some functional motifs in the cytoplasmic region of HIV-1 M Vpus proposed to be important for anti-tetherin activity are more frequently found in the Vpu proteins of SIVcpzPtt than in those of SIVcpzPts infecting eastern chimpanzees (P. t. schweinfurthii), that have not been detected in humans, and SIVgor from gorillas, which is closely related to HIV-1 O and P. Thus, SIVcpzPtt strains may require fewer adaptive changes in Vpu than SIVcpzPts or SIVgor strains to counteract human tetherin.

Results: To examine whether SIVcpzPtt may only need changes in the transmembrane domain (TMD) of Vpu to acquire anti-tetherin activity, whereas SIVcpzPts and SIVgor may also require changes in the cytoplasmic region, we analyzed chimeras between the TMD of an HIV-1 M Vpu and the cytoplasmic domains of SIVcpzPtt $(n=2)$, SIVcpzPts $(n=2)$ and SIVgor $(n=2)$ Vpu proteins. Unexpectedly, all of these chimeras were capable of counteracting human tetherin to enhance virion release, irrespective of the presence or absence of the putative adaptor protein binding sites and the DSGXXS $\beta$-TrCP binding motif reported to be critical for effective anti-tetherin activity of M Vpus. It was also surprising that in three of the six chimeras the gain of anti-tetherin function was associated with a loss of the CD4 degradation activity since this function was conserved among all parental HIV-1, SIVcpz and SIVgor Vpu proteins.

Conclusions: Our results show that changes in the TMD of SIVcpzPtt, SIVcpzPts and SIVgor Vpus are sufficient to render them active against human tetherin. Thus, several previously described domains in the extracellular region of Vpu are not absolutely essential for tetherin antagonism but may be required for other Vpu functions.
\end{abstract}

\section{Background}

In order to replicate and spread efficiently in their respective hosts primate lentiviruses have to counteract a variety of innate host restriction factors that are frequently induced by type I interferons and inhibit viruses at various steps in their life cycle [1,2]. As a countermeasure, primate lentiviruses have evolved effective antagonists that

\footnotetext{
*Correspondence: frank.kirchhoff@uni-ulm.de

'Institute of Molecular Virology, Ulm University Medical Center, 89081, Ulm, Germany

Full list of author information is available at the end of the article
}

counteract these restriction factors, e.g. by targeting them for proteasomal degradation or by sequestering them away from their viral targets $[3,4]$. Both, the antiviral factors and their viral antagonists are under strong selection pressure for diversification [2]. As a consequence, host restriction factors are highly divergent and frequently counteracted by viral factors in a species-specific manner. Thus, they may pose significant hurdles to cross-species transmissions [4].

The genetic barrier for cross-species transmission is reduced between closely related species because pathogens may already be able to evade or counteract some host

\section{Biomed Central}


defense mechanisms. For example, simian immunodeficiency viruses (SIVs) infecting chimpanzees and gorillas that represent the direct precursors of human immunodeficiency virus type 1 (HIV-1) are resistant against human tripartite motif-containing protein 5 (TRIM5 $\alpha$ ) [5], which induces untimely uncoating of retroviral capsids [6,7]. Furthermore, SIVcpz and (most likely) SIVgor Vif are capable of antagonizing the human APOBEC3G orthologue because it is highly homologous to the corresponding ape proteins [8]. Thus, adaptation of these SIVs to our closest non-human relatives has already disarmed two potent human defense factors. In contrast, primate lentiviruses are unable to counteract the human tetherin orthologue because it contains a deletion in the cytoplasmic region that renders it resistant to the accessory protein Nef, which is used by most SIVs (including SIVcpz and SIVgor) to counteract tetherin [9-11]. As a consequence, tetherin that inhibits virus release by tethering nascent virions at the cell surface seems to constitute a significant barrier to the effective spread of primate lentiviruses in humans [12]. Thus far, only pandemic HIV-1 group $\mathrm{M}$ strains have fully cleared this hurdle by the acquisition of effective Vpu-mediated anti-tetherin activity [9]. In comparison, HIV-1 group $\mathrm{O}$ and $\mathrm{P}$ strains, which resulted from independent zoonotic transmissions and are closely related to SIVgor [13,14] have apparently not yet evolved an effective antagonist of human tetherin $[9,15]$. Finally, Vpus of the rare HIV-1 N strains acquired some antitetherin activity in humans, but lost the second key function of $\mathrm{Vpu}$, i.e. degradation of $\mathrm{CD} 4$, the primary viral receptor $[9,16]$.

Molecular epidemiological studies of SIVcpz in wildliving chimpanzees throughout central Africa have shown that only viruses infecting P.t. troglodytes but not $P$. $t$. schweinfurthii apes have crossed the species barrier to humans [17-19], although SIVcpz infection is common in both of them [20]. Furthermore, only the $\mathrm{M}$ and $\mathrm{N}$ groups of HIV-1 that were transmitted from central chimpanzees (Ptt) but not group O and P HIV-1 strains that are most closely related to SIVgor, gained $\mathrm{Vpu}$-mediated anti-tetherin activity during adaptation to humans [9]. The transmembrane domain (TMD) of Vpu seems to interact directly with the TMD domain of tetherin and changes in the TMD were critical for the gain of anti-tetherin activity of group $\mathrm{M}$ and $\mathrm{N}$ viruses during adaptation to humans $[9-11,16]$. Furthermore, it has been reported that certain tyrosine- $(\mathrm{Y} \times x \phi)$ and dileucine-based (E/DxxxLL/I/V/M) sorting motifs in the cytoplasmic domain of $\mathrm{Vpu}$ are critical for effective tetherin antagonism by M Vpus [21-24]. Sequence analyses revealed that these motifs are conserved in most SIVcpzPtt Vpus but absent in SIVcpzPts Vpus [20]. Thus, these findings raised the possibility that the Vpu proteins of SIVcpzPtt strains might only have to acquire alterations in the TMD of Vpu to evolve anti-human tetherin activity, whereas those of SIVcpzPts and SIVgor would also need changes in the cytoplasmic region [20]. To examine this possibility, we generated chimeric proteins between the TMD of the HIV-1 M NL4-3 Vpu and the cytoplasmic regions of SIVcpzPtt, SIVcpzPts and SIVgor Vpus. Unexpectedly, we found that all of these chimeric proteins were potent tetherin antagonists, although some of them lack functional domains thought to be important for potent counteraction of tetherin by group M Vpus.

\section{Results}

In the present study, we examined whether differential adaptive hurdles of SIVcpz Vpu could explain why only one of two infected subspecies of chimpanzees served as a zoonotic reservoir for humans and why HIV-1 groups $\mathrm{O}$ and $\mathrm{P}$ that are most closely related to SIVgor [25] failed to acquire Vpu-mediated anti-tetherin activity (Figure 1A). As shown in Figure 1B, several domains reported to be important for the anti-tetherin activity of HIV-1 $M$ Vpus, such as a putative AP-binding Yxx $\phi$ motif [22,26], D/ExxxLL/I/V/M endocytosis motifs [21] and a DSGxxS $\beta$-TrCP interaction domain [26-28], are preserved in the SIVcpzPtt MB897 and EK505 Vpus, but not in those derived from the SIVcpzPts TAN3 and ANT strains. The SIVgor CP2139 and BQ664 Vpus do not contain a Yxx $\phi$ motif adjacent to their TMD and the DSGxxS domain is changed to DEGxxS (Figure 1B). Notably, it has been shown that a phosphorylated serine residue may be mimicked by an acidic residue [29] suggesting that the latter motif may still be capable of interacting with $ß-\operatorname{TrCP}$. To examine whether the SIVcpzPtt Vpus may only require alterations in the TMD to counteract human tetherin, whereas the SIVcpzPts and SIVgor Vpus may need additional changes in cytoplasmic part (CP), we generated a set of chimeras between the TMD of the HIV-1 M NL4-3 Vpu, which is a well characterized antagonist of human tetherin, and the $\mathrm{CP}$ of these SIV Vpus (Figure 1B; Additional file 1: Table S1). All wild-type and chimeric Vpus were expressed at detectable albeit highly variable levels in transfected 293T and HeLa cells except for the SIVcpzPts TAN3 Vpu (Figure 2A). The latter was difficult to detect by Western blot, although this protein efficiently degrades CD4 [9]. It is known that the functional activity of Vpu proteins does not always correlate with their in vitro expression levels detected by Western blot, possibly because they aggregate and/or remain associated with the insoluble membrane fraction $[9,16]$.

To examine the ability of these wild-type and chimeric $\mathrm{Vpu}$ proteins to reduce cell surface expression of tetherin, we transfected 293T cells with constructs co-expressing the various Vpus and eGFP together with a construct expressing human tetherin and analyzed them by flow cytometry. In the absence of $\mathrm{Vpu}$, cells co-expressed high levels of 

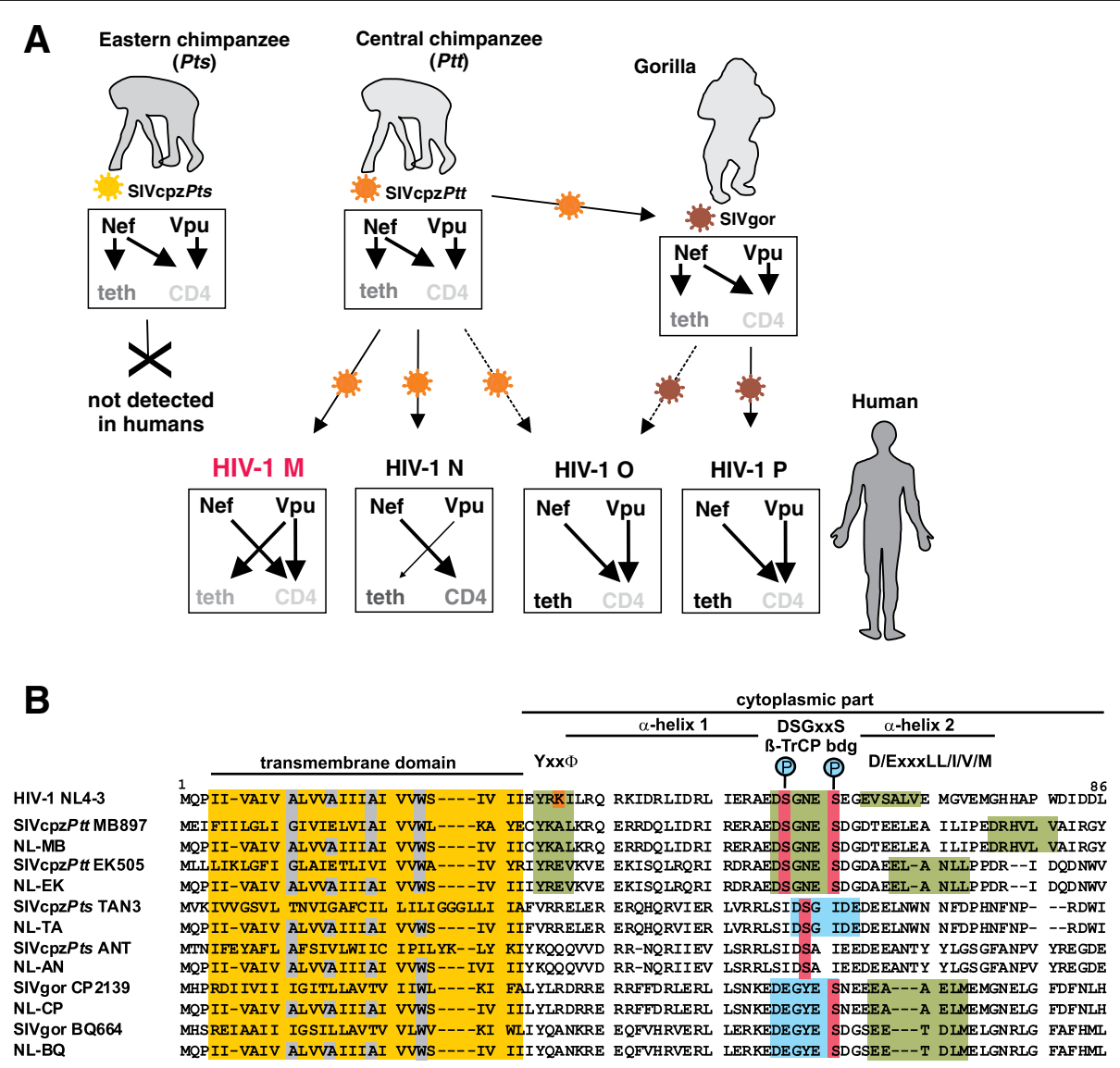

Figure 1 Zoonotic transmissions of SIVs from apes to humans and adaptive changes in Vpu and Nef functions. (A) The Nef proteins of SIVcpz and SIVgor down-modulate CD4 from the cell surface and counteract tetherin in their non-human hosts. Upon cross-species transmission of SIVcpz and SIVgor to humans, Nef-mediated tetherin antagonism was disrupted by a unique deletion in the cytoplasmic tail of the human tetherin orthologue. Subsequently, Vpu evolved to counteract tetherin during the emergence of pandemic HIV-1 M strains. In contrast, HIV-1 O and P Vpus did not gain anti-tetherin activity. Finally, Vpus of group $N$ viruses evolved some activity against human tetherin but lost their ability to degrade CD4. The arrows indicate activity or cross-species transmissions. Grey indicates antagonism of tetherin or CD4 by one and light grey by two viral factors. (B) Alignment of SIVcpz and SIVgor Vpu amino acid sequences. The HIV-1 NL4-3 Vpu sequence is shown on top for comparison. The AxxxAxxxAxxxW residues in the TMD that are important for anti-tetherin activity of M Vpus, a putative Yxx motif, two phosphorylation sites in the DSGxxS B-TrCP interaction site, and an E/DxxxLL/IN/M motif involved in targeting of tetherin for endosomal degradation are indicated. The consensus DSGxxS B-TrCP interaction site is highlighted in green and sites containing mutations of the serines to acidic residues in light blue. Dashes indicate gaps introduced to optimize the alignment.

tetherin and eGFP (Figure 2B). In agreement with previous results [9], the NL4-3 Vpu significantly reduced the levels of tetherin expression at the cell surface, whereas the wild-type SIVcpz and SIVgor Vpus were largely inactive (Figure 2B, 2C). The TMD of the NL4-3 Vpu significantly increased the capability of the MB897 and BQ664 Vpus to reduce the surface levels of tetherin in transfected 293T cells, but had only little (if any) effect on the functional activity of the EK505, TAN3, ANT and CP2139 Vpus (Figure 2B, 2C). To test whether the latter was the result of artificial over-expression of tetherin, we examined the of the various wild-type and chimeric Vpu proteins effects on the surface expression of endogenously produced tetherin in HeLa cells (Figure 2D, Additional file 2: Figure S1). We found that the $\mathrm{Vpu}$-dependent reduction of tetherin surface expression was more pronounced in HeLa than in 293T cells, e.g. the NL4-3 Vpu achieved an 85\% reduction in the former but only $60 \%$ in the latter (Figure 2B-D). This difference in tetherin down-modulation efficiency is most likely a consequence of the higher expression levels in transiently transfected 293T cells (Figure 2E). Unexpectedly, replacement of the SIVcpz and SIVgor Vpu TMD by that of HIV-1 resulted in a reduction of tetherin surface expression in HeLa cells of $60 \%$ to $90 \%$ (Figure 2D). Even more surprisingly, the chimera between NL4-3 and ANT Vpu (NL-AN) showed the highest activity, although it lacks the Yxxp, DSGxxS and E/DxxxLL/I/ $\mathrm{V} / \mathrm{M}$ motifs in the cytoplasmic region (Figure 1B). The 


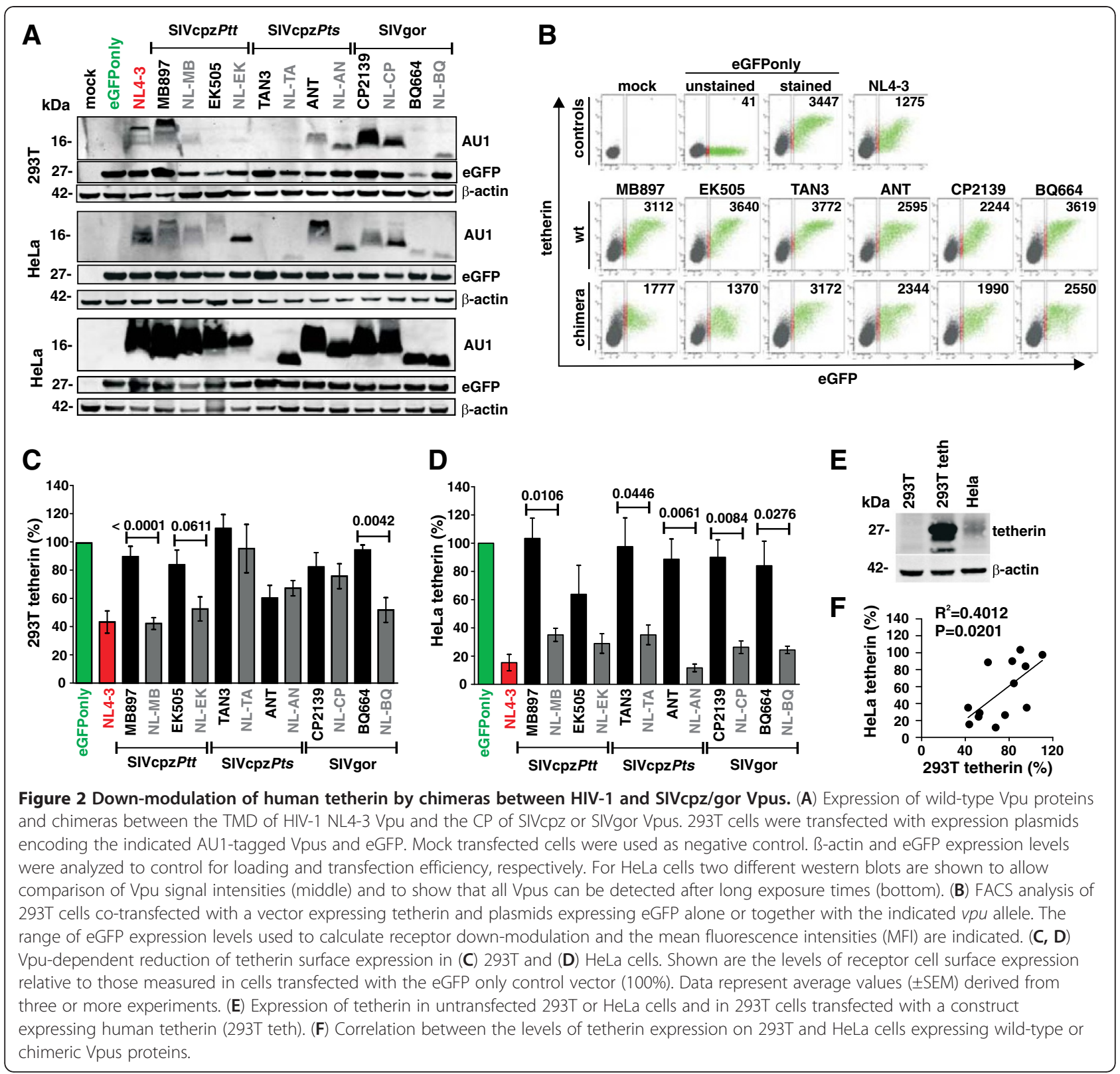

results from 293T and HeLa cells were correlated, albeit imperfectly, because some Vpus were substantially more active in the latter cell type (Figure 2F).

To determine the ability of wild-type and chimeric SIVcpz and SIVgor Vpus to promote virus release in the presence of tetherin, we co-transfected $293 \mathrm{~T}$ cells with a $v p u$ defective HIV-1 NL4-3 construct, pCGCG vectors expressing AU1-tagged versions of $\mathrm{Vpu}$ and different doses of human tetherin. In contrast to the parental SIVcpz and SIVgor proteins, all chimeras were capable of promoting infectious virus release (Figures 3A). The chimera between the HIV-1 TMD and the SIVgor BQ664 CP (NL-BQ) increased infectious virus release in both $293 \mathrm{~T}$ and HeLa cells almost as efficiently as the control NL4-3 Vpu and was substantially more effective than the chimeras between HIV-1 and SIVcpzPtt Vpus (NL-MB, NL-EK) (Figures 3A, 3B). This was surprising because in contrast to the SIVcpzPtt MB897 and EK505 Vpus, the cytoplasmic part of the SIVgor BQ664 Vpu contains substitutions in the DSGxxS motif (DEGYES) and lacks a putative $\operatorname{Yxx} \phi$ motif (Figure 1B). These results were confirmed by measuring the quantity of fectious virus and p24 release in the culture supernatant of HeLa cells co-transfected with proviral HIV-1 and $\mathrm{Vpu}$ expression constructs (Figures $3 \mathrm{C}$ to E). All Vpus that promoted infectious virus release in transiently transfected 293T cells were also active in HeLa cells (Figure 3F) and most $\mathrm{Vpu}$ proteins that reduced 

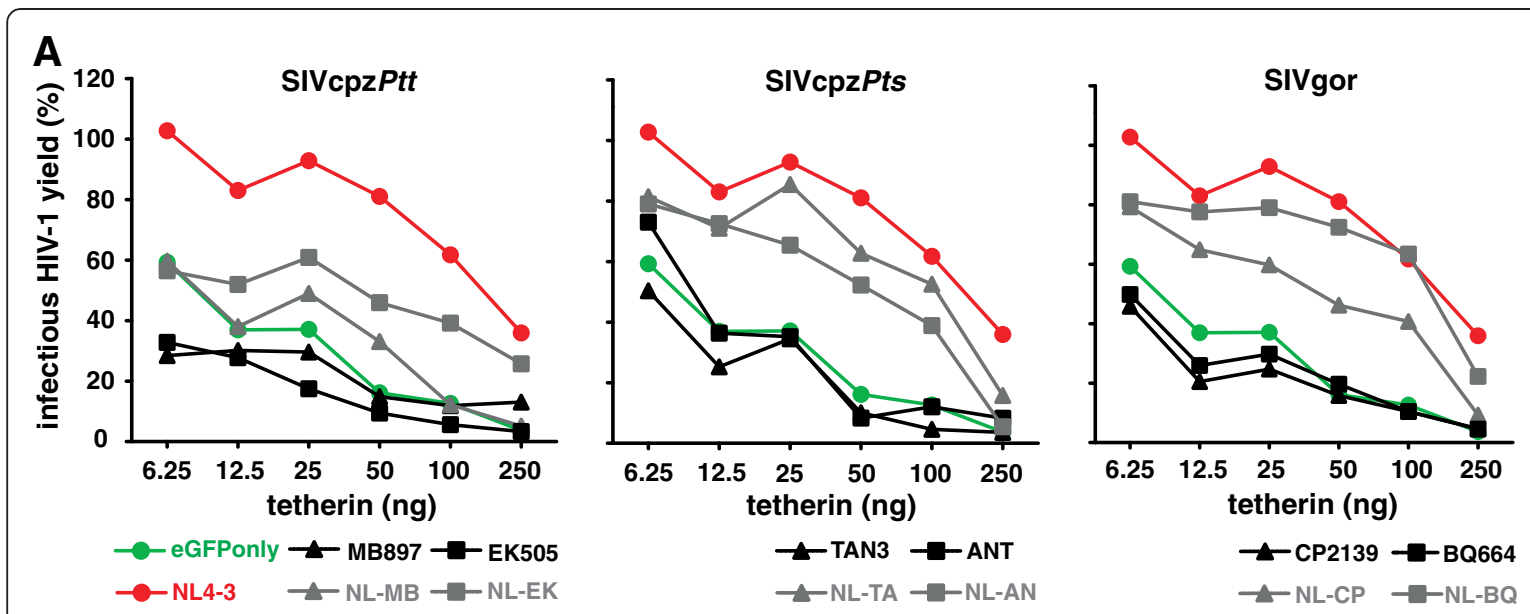

B

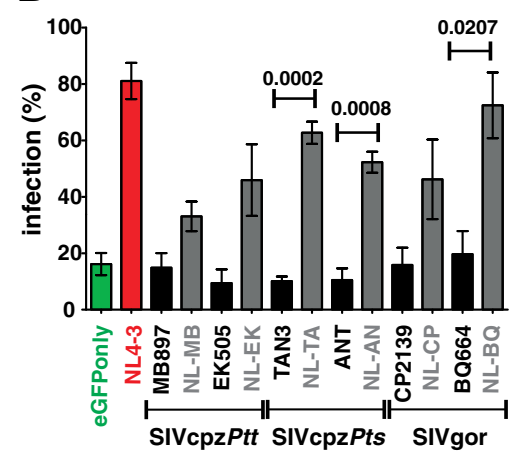

C

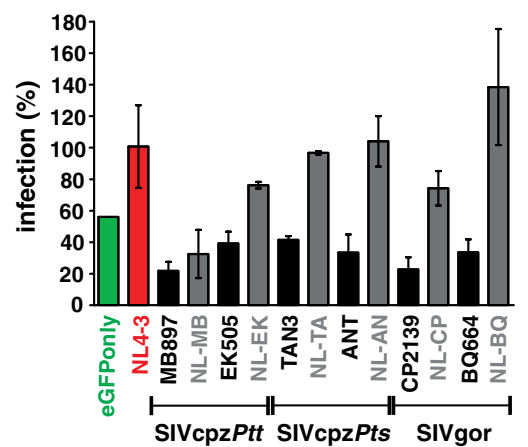

D
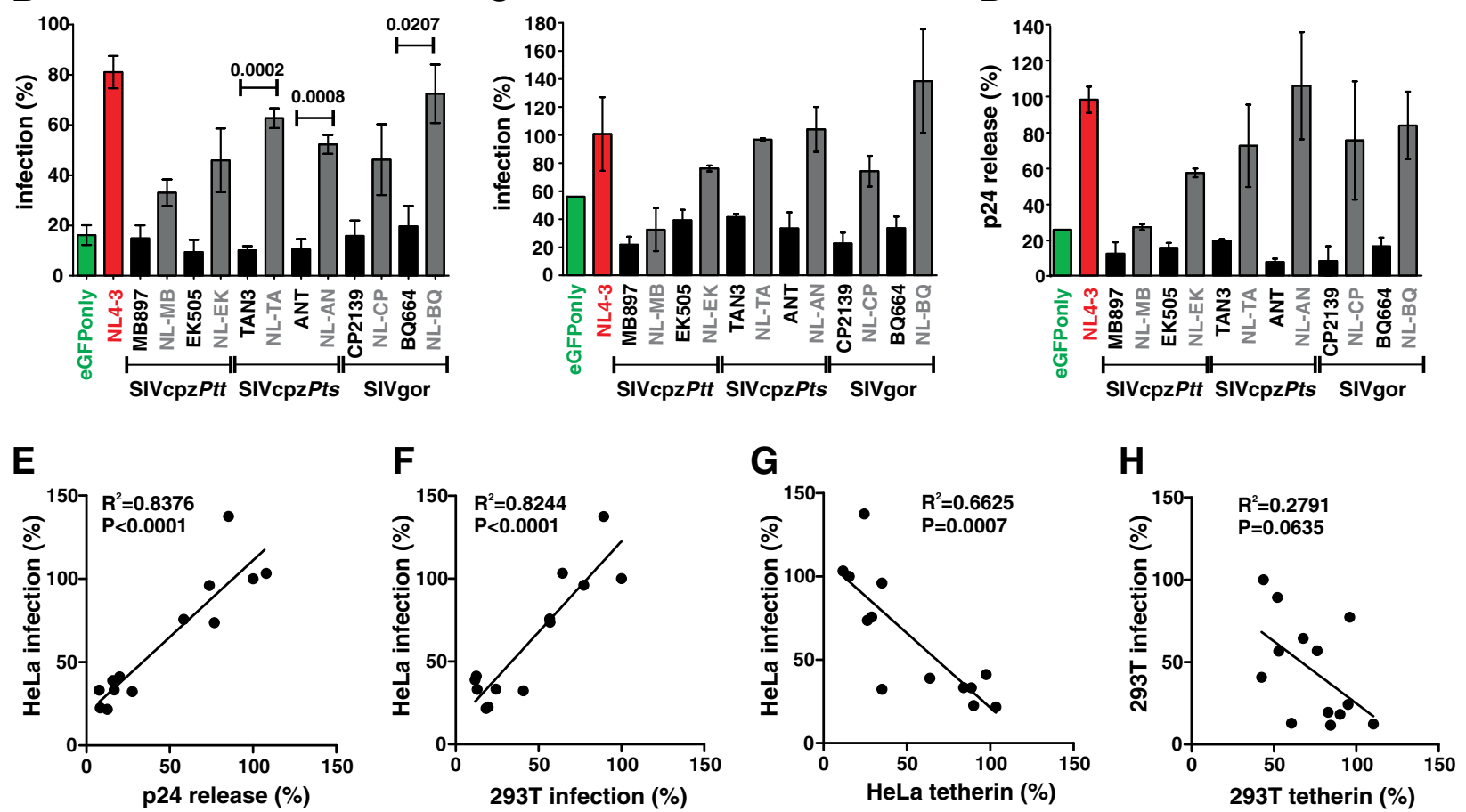

Figure 3 Enhancement of virus release in the presence of human tetherin by chimeras between HIV-1 and SIVcpz/gor Vpu. (A) Infectious virus yield from 293 T cells co-transfected with an HIV-1 NL4-3 $\Delta v p u$ construct and vectors expressing the indicated vpu alleles in combination with various quantities of plasmids expressing tetherin. Shown are average values derived from triplicate infections of TZM-bl indicator cells relative to those obtained in the absence of tetherin (100\%). The mean of three independent experiments is shown. The results were verified by measuring the cell-free p24 levels by ELISA. (B) Infectious virus yield from 293T cells co-transfected with the proviral HIV-1 NL4-3 $\Delta v p u$ construct $(4 \mu \mathrm{g})$, pCGCG vectors expressing the indicated vpu alleles (1 $\mathrm{ng})$ and a plasmid expressing human tetherin (50 $\mathrm{ng}$ ). Shown are average values ( \pm SEM) derived from three independent experiments each involving triplicate infections of TZM-bl indicator cells. (C, D) Detection of (C) infectious HIV-1 and (D) p24 antigen in the supernatant of HeLa cells that express tetherin endogenously and were co-transfected with HIV-1 NL4-3 $\Delta v p u$ and the indicated Vpu expression constructs. Data represent average values ( \pm SEM) derived from three experiments. (E-G) Correlation between infectious virus release from HeLa cells and (E) p24 release, (F) infectious virus release from 293 T cells and (G) tetherin cell surface expression levels of HeLa cells. (H) Correlation of infectious virus release and tetherin surface expression levels of $293 \mathrm{~T}$ cells.

tetherin surface expression also enhanced virion release (Figure 3G, 3H).

To further analyze the unexpected activity of the chimeric Vpus against human tetherin, we next performed microscopic analyses in HeLa cells to examine their effect on the subcellular localization of this restriction factor. In the absence of $\mathrm{Vpu}$ and in cells expressing wild-type SIVcpzPtt, SIVcpzPts and SIVgor Vpu proteins, tetherin co-localized with the trans-Golgi network (TGN) marker TGN46 but was also present at the cell surface (Figure 4). 


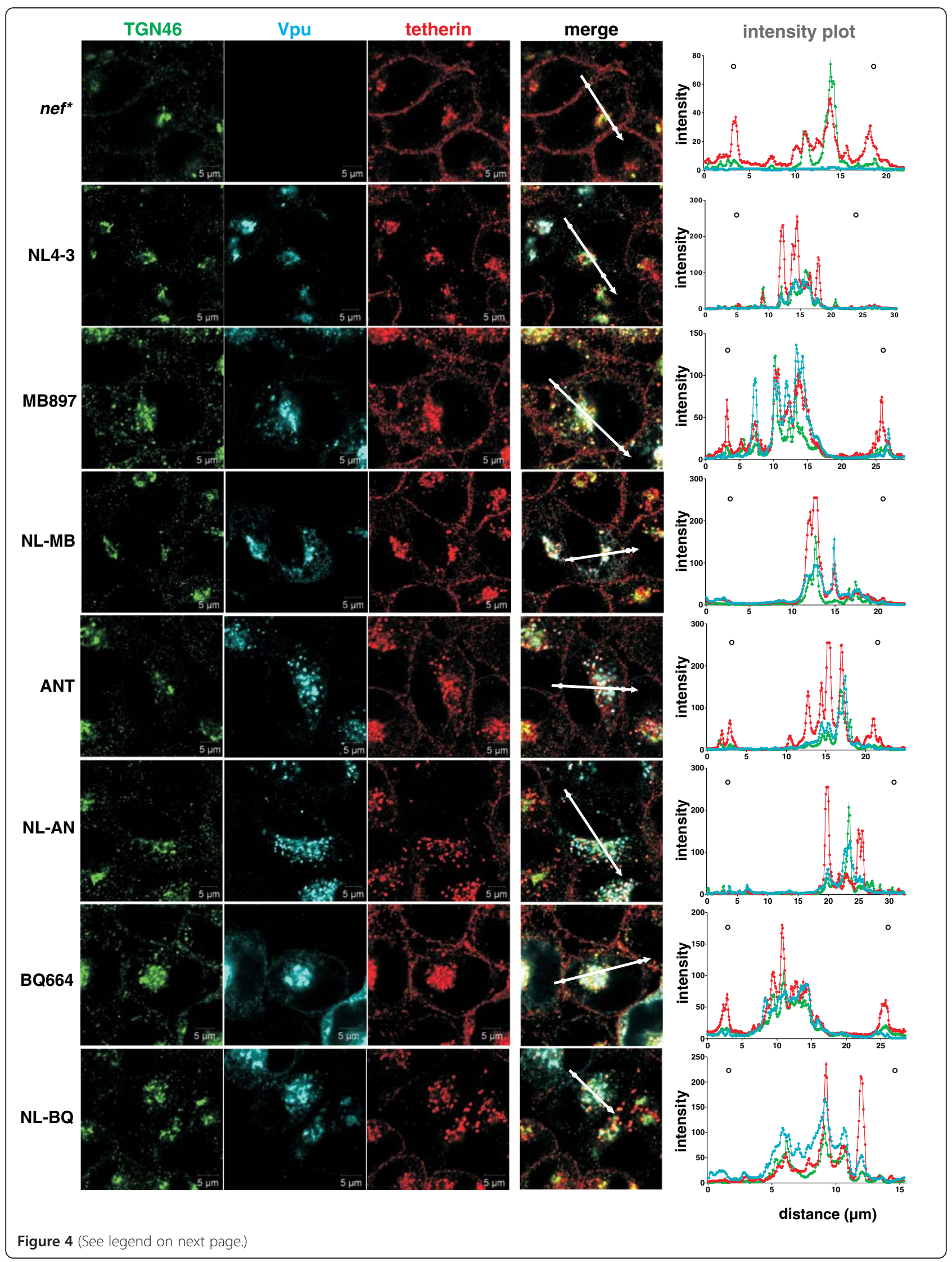


(See figure on previous page.)

Figure 4 Cellular localization of tetherin in the presence of various Vpu proteins. Confocal immunofluorescence images of HeLa cells transfected with constructs expressing the indicated Vpu variants. Two days post-transfection, cells were fixed and permeabilized for intracellular staining of tetherin (red), Vpu (blue) and the TGN (green). Images show representative confocal acquisitions of at least 40 transfected cells investigated. Tetherin was located at the cell surface in cells transfected with constructs expressing the parental SIVcpz and SIVgor Vpus but not in cells expressing chimeras containing the TMD of the NL4-3 Vpu. Cellular localization was determined by microscopic examination and by analysis of the Vpu, tetherin and TGN signal intensities throughout the cells (right panel). The regions utilized to generate the profile plots are indicated by the arrows. White circles indicate the position of the plasma membrane.

In contrast, all Vpu chimeras prevented tetherin expression at the cell surface as effectively as the wild-type NL4-3 Vpu (Figure 4). Thus, the results of the microscopic analyses confirmed that the HIV-1 TMD SIVcpz/gor CP chimeras are effective tetherin antagonists that promote effective virion release by sequestering the restriction factor away from the cell surface. Our results further suggest that co-localization of $\mathrm{Vpu}$ and tetherin in the TGN may be required but not sufficient for tetherin antagonism.

Another well established function that is conserved among all primate lentiviral Vpus, except for those of HIV-1 group $N$ strains $[9,16]$, is the ability to degrade the CD4 receptor [30-32]. To determine whether the Vpu chimeras were active against CD4, we co-transfected $293 \mathrm{~T}$ cells with vectors co-expressing Vpu and eGFP (or eGFP alone for control) together with a CD4 expression construct. In the absence of $\mathrm{Vpu}$, cells expressed high levels of CD4 and eGFP (Figure 5A, Additional file 3: Figure S2). All wild-type HIV-1, SIVcpz and SIVgor Vpu proteins markedly reduced CD4 cell surface expression, whereas several Vpu chimeras were poorly active (Figure 5A). Quantitative analyses showed that the parental Vpus usually reduced CD4 cell surface expression by more than $80 \%$, whereas the NL-MB, NL-EK and NL-BQ chimeras only achieved $20 \%$ to $50 \%$ reduction (Figure $5 \mathrm{~B}$ ). Notably, the chimeras between the HIV-1 Vpu TMD and the SIVcpzPts TAN3 and ANT as well as SIVgor CP2139 CPs of $\mathrm{Vpu}$ maintained most of their anti-CD4 activity (Figure 5B). Analysis of HeLa cells transfected with CD4 expression constructs and of TZM-bl cells stably expressing CD4 (Additional file 3: Figure S2) confirmed these results (Figure $5 \mathrm{C}-\mathrm{F}$ ). The effects were generally weaker in TZM-bl cells than in transiently transfected 293T and HeLa cells (Figure 5B-D). This is most likely due to the fact that $\mathrm{Vpu}$ mediates efficient degradation of intracellular CD4 but is unable to remove CD4 molecules from the surface that were already at the plasma membrane prior to transfection [27,30-32]. In 293T cells, we observed a significant inverse correlation between the effect of the panel of $\mathrm{Vpu}$ protein analyzed on tetherin and CD4 surface expression (Figure 5G). The same trend was found in HeLa cells but failed to reach significance (Figure $5 \mathrm{H}$ ) because the NL-AN and NL-CP chimeras reduced the surface expression of both receptors (Figures 2D and $5 \mathrm{C}$ ). These results suggest that the acquisition of changes in the TMD of $\mathrm{Vpu}$ that confer anti-tetherin activity may have detrimental effects on the CD4 degradation activity in the context of some SIVcpz and SIVgor vpu alleles.

$\mathrm{Vpu}$ interacts with the cytoplasmic part of CD4 and binds 3 -TrCP to recruit an E3 ubiquitin ligase complex to CD4 thereby inducing its degradation by cellular proteasomes [27]. Effective CD4 degradation by M Vpus requires a functional DSGxxS $\beta$-TrCP target motif, where the serines need to be phosphorylated to allow efficient recruitment of $\beta$-TrCP $[31,32]$. We have previously shown that SIVcpz and SIVgor Vpus are highly active against CD4 [9], although the DSGxxS motif is frequently altered (e.g. to DSAIEE or DEGYES) and contains only a single putative phosphorylation site (Figure 1B). To examine whether the wild-type and chimeric Vpus are capable of interacting with $ß$-TrCP, we fused the $\mathrm{N}$-terminal fragment of the click beetle luciferase to the $\mathrm{C}$-terminus of $\mathrm{Vpu}$, and the $\mathrm{C}$-terminal fragment of this luciferase to the $\mathrm{N}$-terminus of $\mathrm{B}$ - $\mathrm{TrCP}$ (Figure 6A). If $\mathrm{Vpu}$ and $ß-\mathrm{TrCP}$ interact, functional luciferase is assembled [33]. The interaction of $B$-catenin and $B$ - TrCP served as positive control [33]. We found that the wild-type HIV-1 and SIVcpz or SIVgor Vpus interacted with $\beta$-TrCP, although the detectable levels of luciferase reporter activity varied (Figures 6A, Additional file 4: Figure S3A). In agreement with published data $[28,32,34,35]$, mutations in the DSGxxS motif of the NL4-3 Vpu (S52A/S56A or G53D) significantly reduced this interaction. The ANT and BQ664 Vpus that contained alterations in the $\beta-\operatorname{TrCP}$ binding site showed weaker interaction than the MB897 Vpu (Figure 6A, Additional file 4: Figure S3A), which has the consensus DSGxxS motif. However, all of them were highly effective in reducing CD4 cell surface expression (Figure 5). Most importantly, exchanges of the TMD had no significant effect on the interaction of $\mathrm{Vpu}$ with $\mathrm{B}-\mathrm{TrCP}$ (Figure 6A, Additional file 4: Figure S3A). Thus, reduced $ß-T r C P$ binding was not the reason for the reduced anti-CD4 activity of some chimeric Vpus.

Next, we used the click beetle complementation assay to examine whether replacement of the TMD of SIVcpz or SIVgor Vpus may affect their ability to bind CD4. We found that all Vpus resulted in increased (albeit variable) levels of luciferase reporter activity compared to ß-catenin, which has not been shown to interact with CD4. This 


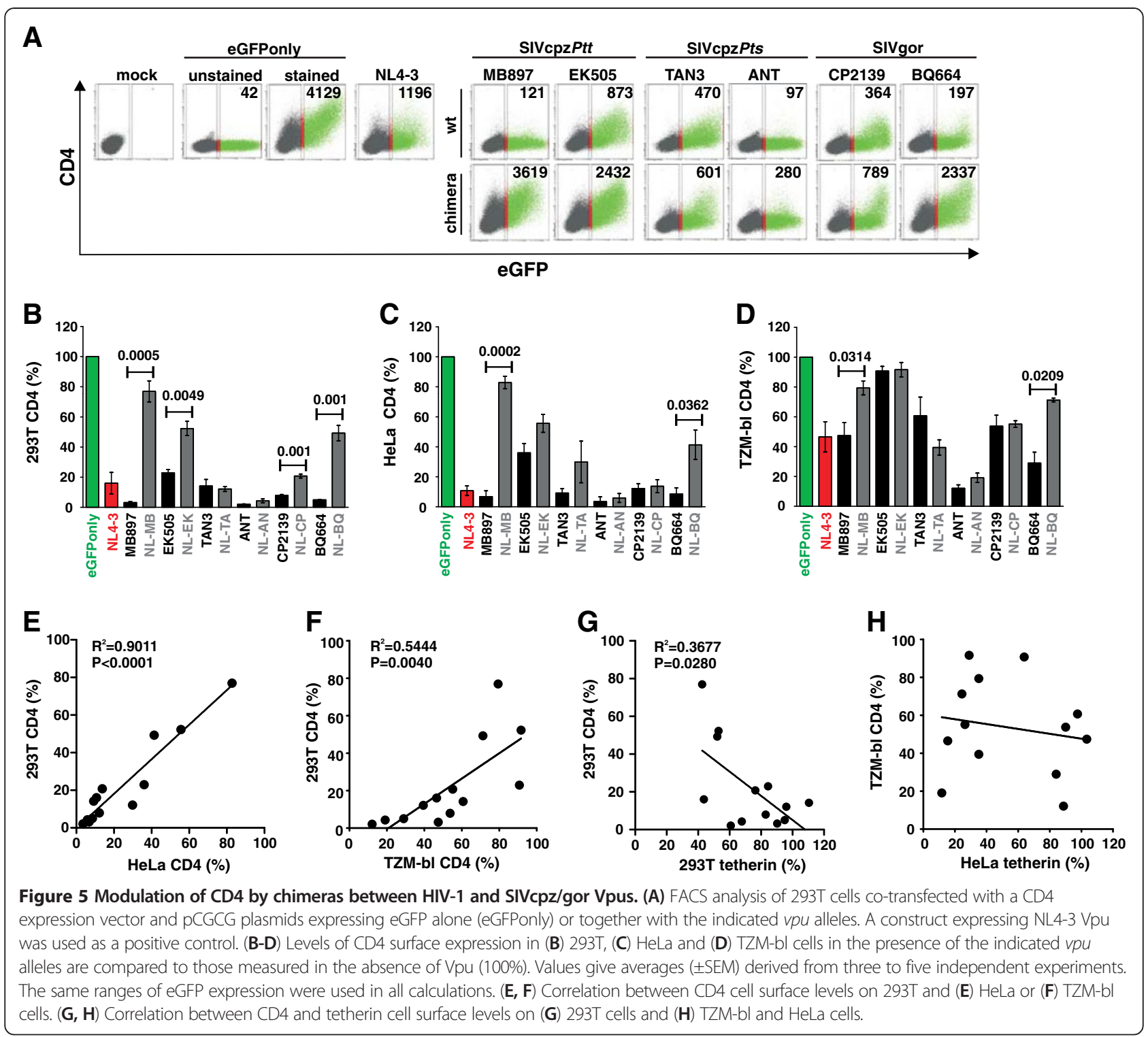

suggests that all Vpus are capable of interacting with CD4 (Figure 6B, Additional file 4: Figure S3B). Mutations in the ß-TrCP binding site of the NL4-3 Vpu increased the signal intensities because the binding of $ß-\mathrm{TrCP}$ and subsequently CD4 luciferase reporter construct degradation was impaired (Figure 6B, Additional file 4: Figure S3B). Replacement of the TMDs of the MB897, ANT and BQ664 Vpus by that of the NL4-3 Vpu had differential and modest effects on luciferase activity. To examine whether some of these differences were the result of an altered interaction or Vpu-mediated degradation of the CD4 luciferase reporter construct, we repeated the interaction assay in the presence of a trans-dominant negative mutant of $\beta$-TrCP1 (TrCP1 $\triangle$ Fbox). This mutant still binds Vpu via WD repeats but lacks the Fbox, and thus fails to recruit the E3 ubiquitin ligase complex for proteasomal degradation of CD4 [32]. In the presence of the $\operatorname{TrCP} 1 \Delta$ Fbox mutant, all $\mathrm{Vpu}$ chimeras resulted in similar or moderately increased (NL-MB) levels of luciferase reporter activities compared to the respective wild-type SIVcpz and SIVgor Vpus proteins (Figure 6C, S3C) suggesting that the exchange of the TMD motif did not disrupt CD4 binding. Thus, the NL-MB and NL-BQ Vpu chimeras were poorly active in reducing CD4 cell surface expression (Figure 5), although they were efficiently expressed (Figure 2A) and capable of interacting with both $ß-\operatorname{TrCP}$ and CD4 (Figure 6, Additional file 4: Figure S3).

\section{Discussion}

In the present study, we show that the $\mathrm{Vpu}$ proteins of six SIVcpzPtt, SIVcpzPts and SIVgor strains became antagonists of human tetherin when their TMD was 

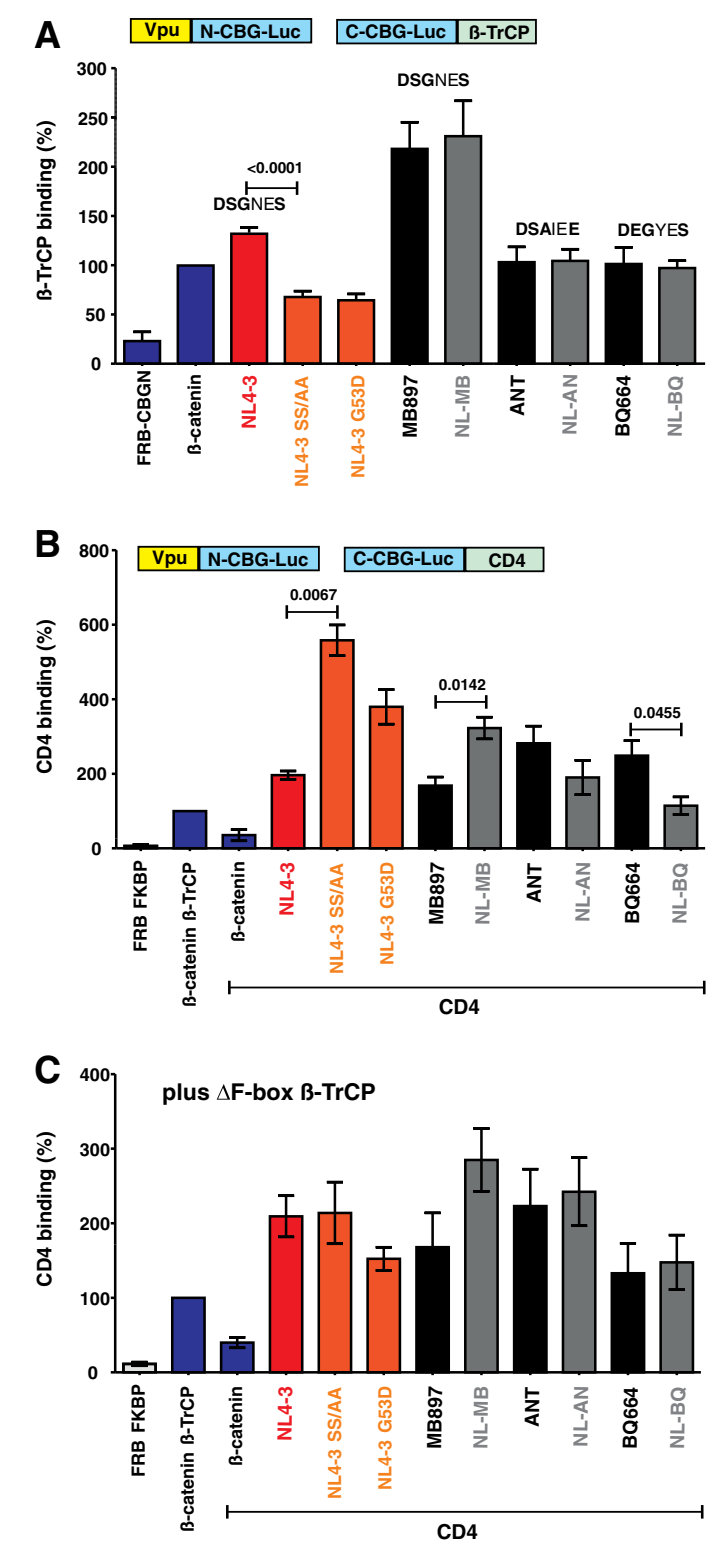

Figure 6 Interaction of chimeras between HIV-1 and SIVcpz/gor Vpus with B-TrCP and CD4. (A) Interaction of Vpu with B-TrCP. 293T cells were transfected with equal amounts of plasmids expressing $B-\operatorname{TrCP}$ $\mathrm{N}$-terminally fused to the C-terminal fragment of click beetle green (CBG) and Vpu C-terminally fused to the $\mathrm{N}$-terminal fragment of CBG. B-catenin served as positive control. After $48 \mathrm{~h}$, click beetle luciferase activity was determined in living cells by addition of D-luciferin and quantification of bioluminescence. Values represent means $( \pm$ SEM) derived from five independent experiments. (B) Interaction of Vpu with CD4. 293T cells were transfected with plasmids expressing CD4 C-terminally fused to the C-terminal fragment of CBG and Vpu C-terminally fused to the Nterminal fragment of CBG and interaction efficiencies were determined as described in the methods section. (C) To exclude a bias caused by Vpu-mediated degradation of CD4, a dominant negative mutant of $\beta$-TrCP1 isoform 2 lacking the F-box (amino acids 141-193) was co-transfected in a control experiment. This mutant still binds Vpu but lacks the Fbox domain and thus fails to recruit the E3 ubiquitin ligase complex. Values represent means ( $₫$ SEM) derived from three to four independent experiments. replaced by that of the HIV-1 M NL4-3 Vpu. The TMD domain of HIV-1 Vpu interacts with human tetherin $[24,34,36]$ and recent data suggest that this interaction is sufficient for some anti-tetherin activity [16,21,26]. Efficient counteraction of tetherin by M Vpus, however, also requires several cytoplasmic domains [21,22,26,28,35]. It has been suggested that the absence of these motifs contributes to the lack of anti-tetherin activity of O Vpus [26] and may explain the poor activity of group $\mathrm{N}$ Vpus against human tetherin [16]. The Vpu proteins of many SIVcpz and SIVgor strains, in particular those from SIVcpzPts strains, lack the canonical functional motifs. Thus, it came as a surprise when the majority of chimeric Vpus were almost as effective in reducing cell surface expression of tetherin and in promoting virus release as the group M NL4-3 Vpu. Even more strikingly, the most active chimeras were not the ones between HIV-1 and SIVcpzPtt Vpus that contained the canonical Yxx $\phi, E / D x x x L L / I / V / M$ and DSGxxS $\beta$-TrCP binding motifs, but those between HIV-1 and SIVcpzPts or SIVgor Vpus that lacked most or all of these putative domains [20] (Figure 1B). These results show that the presence or absence of "binding motifs" important for effective tetherin antagonism by $M$ Vpus cannot be used to predict the functionality of the cytoplasmic domain of SIVcpz and SIVgor Vpus. The data also suggest that most SIVcpz and SIVgor Vpus, regardless of their genetic diversity, require only changes in their TMD to become active against human tetherin.

Since SIVcpz and SIVgor clearly use Nef to counteract the tetherin restriction in their natural ape hosts [9], it may seem striking that the cytoplasmic domains of their $\mathrm{Vpu}$ proteins fulfill the requirements to antagonize tetherin. The $v p u$ gene is believed to have originated in a precursor of SIVs that infects guenons (SIVgsn/mus/mon), which subsequently recombined with an SIV strain that infects red-capped mangabeys (SIVrcm) to generate SIVcpz [37,38]. The Vpus from current SIVgsn, SIVmus and SIVmon strains are all capable of counteracting the tetherin orthologue of their cognate host species [9]. Thus, the Vpu that SIVcpz received from the precursor of the SIVgsn/mus/mon lineage was most likely a functional tetherin antagonist. However, due to sequence variations in the transmembrane portion of $\mathrm{Vpu}$, tetherin is counteracted in a species-specific manner and subsequently Nef and not Vpu evolved to become an effective tetherin antagonist in chimpanzees [9]. It is tempting to speculate that the cytoplasmic domain of Vpu maintained the requirements for counteracting tetherin because the sequence motifs in $\mathrm{Vpu}$ that enable proper subcellular localization and/or are involved in the recruitment of the ubiquitination/degradation machinery are also critical for CD4 degradation and (possibly) for other Vpu activities, such as down-modulation of the natural killer cell ligand NTB-A and the lipid-antigen presenting protein CD1d 
[39-41]. It is conceivable that domains that are beneficial for several functions will be preserved even if one function is lost. Indeed, recent data have shown that just two amino acid changes are sufficient to render HIV-1 Nef active against chimpanzee tetherin, suggesting that other functional constraints keep Nef "ready" to regain this function [42].

The results of the present study further illustrate the functional plasticity of the viral accessory proteins. It seems clear that several domains that may be critical for full activity of $M$ Vpus are not required for effective tetherin antagonism by chimeric Vpu proteins. Thus, the functional relevance of some cytoplasmic domains in $\mathrm{Vpu}$ seems to be context dependent. Similarly, we have previously observed that some SIV or HIV-2 Nef proteins are fully functional, although they lack some of the domains reported to be critical for HIV-1 Nef function $[43,44]$. It is often difficult to assess whether the required functional interactions with cellular factors are performed by different regions of the viral proteins or whether the sequence variations are tolerated such that the mutated domains remain active. For example, it has been reported that phosphorylation of both serines in the HIV-1 DSGxxS domain is essential for Vpu-mediated degradation of CD4 [45]. However, a phosphorylated serine residue may be mimicked by an acidic residue [29]. For example, SIVgor Vpus that contain a DEGxxS domain interact with $ß-\mathrm{TrCP}$ and downregulate CD4 [9]. Furthermore, some $\mathrm{Vpu}$ proteins that do not contain the Yxx $\phi$ and E/DxxxLL/I/V/M motifs in their cytoplasmic region may use different domains to interact with cellular trafficking or endocytosis motifs. For example, a NPxY (or FxNPxY or FxNPxF) motif is found in many SIVcpz and most HIV-1 O Vpus. It is known that this tyrosine motif allows other type I transmembrane proteins (e.g. the LDL and insulin receptors) to recruit AP-2 [46-48], but its relevance for $\mathrm{Vpu}$ function remains to be investigated. In either case, our results indicate that the presence or absence of specific sequence motifs in lentiviral accessory proteins do not necessarily predict their functional integrity.

Our results show that it is difficult to predict the changes required for a gain of anti-tetherin activity of SIVcpz or SIVgor Vpus based on results obtained using $M$ Vpus because the effect of sequence alterations is often context dependent. While our data indicate that SIVcpzPtt, SIVcpzPts and SIVgor Vpus may require mainly mutations in their TMDs to acquire significant anti-tetherin activity, they do not allow to estimate which and how many amino acid substitutions are required for optimal adaptation. For example, the adaptive changes in the TMD that allow HIV-1 group $\mathrm{M}$ and $\mathrm{N}$ Vpus are overlapping, but distinct $[16,49]$. Thus, our findings do not refute the previously suggested possibility that SIVcpzPtt may on average require fewer adaptive changes than SIVcpzPts strains to become functional in humans [20].

To avoid possible artifacts due to over expression of tetherin, we examined the effects of $\mathrm{Vpu}$ in both transiently transfected 293T cells and in HeLa cells, which express tetherin endogenously. In HeLa cells, the effects of the various wild-type and chimeric Vpus on the reduction of tetherin cell surface expression and virus release correlated significantly (Figure 3G). However, the effects of $\mathrm{Vpu}$ on cell surface expression of tetherin were weaker in 293T cells and some $\mathrm{Vpu}$ chimeras clearly reduced tetherin surface expression in HeLa cells, but failed to exert a significant effect in 293T cells (Figure 2C, 2D). The reasons for these discrepancies remain unknown, but may involve higher expression levels (Figure 2E) as well as different glycosylation patterns of tetherin. Nonetheless, these results suggest that potent anti-tetherin effects can be monitored in 293T cell-based over-expression assays, but weaker activities against tetherin may be missed.

In some cases, a gain of anti-tetherin activity by the Vpu chimeras was associated with a significant loss of the CD4 degradation function. This came as a surprise because all parental Vpus were active against CD4 and the chimeras thus contained all functional domains required for this activity. The inverse correlation between both functions was dependent on the context of the specific SIVcpz or SIVgor vpu alleles utilized to generate the Vpu chimeras. Specifically, chimeras between the TMD of HIV-1 Vpu and the CP of SIVcpzPtt MB897 and EK505 or SIVgor BQ664 Vpu (NL-MB, NL-EK, NL-BQ) were poorly active against $\mathrm{CD} 4$, whereas the remaining three chimeras (NL-TA, NL-AN and NL-CP) potently suppressed CD4 cell surface expression (Figure 5). Thus, the acquisition of TMD changes facilitating tetherin interaction and counteraction can come at a cost, i.e., the loss in the CD4 degradation function. It remains a matter of speculation whether this may explain the loss of the CD4 degradation activity of group $\mathrm{N}$ Vpus [9]. The reasons for the loss of CD4 down-regulation function in some $\mathrm{Vpu}$ chimeras also remain to be determined as they contain all known interaction domains and bind both CD4 and $ß$-TrCP molecules (Figure 6). Furthermore, efficient expression and anti-tetherin activity of these Vpu chimeras argue against major structural defects. It thus seems clear that the TMD and CP of Vpu must interact in some way for full functionality of the protein.

\section{Conclusions}

A better understanding of the adaptive barriers that primate lentiviruses have to overcome to spread efficiently in humans is essential to assess the potential risk of future pandemics and may help to develop new preventive strategies. Previous results suggest that effective tetherin 
antagonism may have been a prerequisite for the effective spread of pandemic HIV-1 M strains [12,50] and that easier adaptability of $\mathrm{Vpu}$ to humans may explain why only certain SIVcpz and SIVgor strains have become new human pathogens [20]. We show here that Vpus from all major SIVcpz and SIVgor lineages require only changes in their TMD to acquire activity against human tetherin. Thus, these ape viruses seem to be more prone to acquire anti-tetherin activity in humans than previously anticipated and it is thus surprisingly that group $\mathrm{O}$ and $\mathrm{N}$ Vpus have not yet evolved anti-tetherin activity during adaptation to humans. One possible reason for this is that these changes may compromise the CD4 degradation function of $\mathrm{Vpu}$, which may help to explain the loss of the latter activity during adaptation of HIV-1 group $\mathrm{N}$ to humans. Our results illustrate that the plasticity and multi-functionality of primate lentiviral accessory proteins makes reliable predictions concerning their zoonotic potential difficult. Notably, the same multi-functionality may also allow primate lentiviruses to acquire new antagonisms, because functions for endocytosis or proteasomal degradation are already in place.

\section{Methods}

\section{Expression vectors and proviral constructs}

Bi-cistronic CMV-promoter-based pCGCG vectors coexpressing $\mathrm{Vpu}$ or tetherin and the enhanced green fluorescent protein (eGFP) or dsRed2, respectively, have been described previously [9]. The $v p u$ alleles were not codon optimized. Splice-overlap-extension PCR was used to introduce an AU1-tag as well as XbaI and $M l u I$ restriction sites flanking the reading frames. The PCR fragments were cloned into the PCGCG vector using standard cloning techniques. To inhibit $\beta$-TrCP mediated recruiting of the E3 ubiquitin ligase to $\mathrm{Vpu}$, a dominant negative mutant of $\beta$-TrCP1 isoform 2 lacking the Fbox (amino acids 141-193) was synthesized (Genscript, Piscataway, USA) and cloned into the pCGCG vector co-expressing dsRed2 via an IRES [16]. All PCRderived inserts were sequence confirmed. A vpu deleted mutant of a CCR5-tropic HIV-1 NL4-3 proviral derivative [51] was used to determine the effect of various Vpus on infectious virus release following complementation in trans.

\section{Cell culture}

293T and HeLa cells were maintained in Dulbecco modified Eagle medium (DMEM) supplemented with 10\% heat-inactivated fetal bovine serum, $350 \mu \mathrm{g} / \mathrm{ml} \mathrm{L}$-glutamine, $120 \mu \mathrm{g} / \mathrm{ml}$ streptomycin sulfate and $120 \mu \mathrm{g} / \mathrm{ml}$ penicillin. TZM-bl cells, a HeLa cell line derivative that expresses large amounts of CD4, CCR5 and CXCR4 and contain the $B$-galactosidase gene under the control of the HIV-1 promoter [52-54] were kindly provided by Drs. Kappes and $\mathrm{Wu}$ and Tranzyme Inc. through the NIH AIDS Reagent
Program and were kept in DMEM supplemented as described above.

\section{Western blot}

To monitor Vpu expression, HeLa cells were transfected with $5 \mu \mathrm{g}$ of the expression constructs. Two days posttransfection cells were harvested, lysed in M-PER buffer (ThermoScientific) containing 1\% SDS and a protease inhibitor cocktail (Roche) and cell lysates were separated in $10 \%$ SDS-PAA gels in a Tris-Tricine buffer system. After gel electrophoresis, proteins were transferred onto PVDF membranes and probed with anti-AU1 antibody (Covance, MMS-130P). For internal controls, blots were incubated with antibodies specific for eGFP (290-50, Abcam) and $\beta$-actin (8227-50, Abcam). Subsequently, blots were probed with anti-mouse or anti-rabbit IRDye Odyssey antibodies (926-32210, 926-32221) and proteins detected using a LI-COR Odyssey scanner.

\section{Flow cytometric analysis}

To determine the effect of Vpu on tetherin and CD4 cell surface expression, 293T were transfected in 6-well plates by the calcium phosphate method as described previously [55] and HeLa cells using Lipofectamine LTX reagent (Invitrogen) with $1 \mu \mathrm{g}$ of a tetherin (for $293 \mathrm{~T}$ cells) or CD4 (for 293T and HeLa cells) expression vector and $5 \mu \mathrm{g}$ of pCGCG constructs expressing eGFP alone (as control) or together with $\mathrm{Vpu}$. Two days posttransfection, tetherin or CD4 expression was examined by FACS analysis, as described previously [56]. An allophycocyanin (APC)-conjugated anti-human CD4 antibody (Invitrogen; MHCD0405) was used for staining of surface CD4. For staining of surface tetherin an unconjugated anti-tetherin antibody (eBioscience) and an APC-conjugated secondary anti-mouse antibody (Invitrogen, A865) were used. Fluorescence of stained cells was detected by two-color flow cytometry and Vpu-mediated tetherin or CD4 down-modulation was calculated as described previously for the functional analysis of nef alleles [37]. Briefly, for exogenously expressed proteins, the mean fluorescence intensity (MFI) obtained for cells transfected with the control construct expressing only eGFP was compared to the MFI obtained for cells co-expressing Vpu and eGFP to determine the efficiency of tetherin or CD4 downregulation. For endogenously expressed proteins, the MFI of untransfected cells (eGFP negative) was compared to the MFI of transfected cells (eGFP positive). The transfection of the control construct expressing only eGFP served as reference.

\section{Virus release assays}

To determine the capability of $\mathrm{Vpu}$ to antagonize tetherin, 293T or HeLa cells were seeded in six-well 
plates and transfected with $4 \mu \mathrm{g}$ of NL4-3 $\Delta v p u$ IRES eGFP, $1 \mu \mathrm{g}$ Vpu expression plasmid and (for 293T cells) different amounts of a tetherin expression vector $(6.25,12.5,25,50,125$ and $250 \mathrm{ng})$. Two days posttransfection, supernatants were harvested and analyzed for infectious virus release by a 96-well infection assay on TZM-bl indicator cells [55] and the release of p24 antigen by ELISA as described previously [9].

\section{Microscopy}

HeLa cells were transfected using Lipofectamine LTX Reagent (invitrogen) with pCGCG Vpu_AU1 constructs and stained 24 hours post-transfection. Briefly, cells were fixed with 4\% PFA, blocked with BSA and surface tetherin was stained with an anti-tetherin antibody (eBioscience) and a secondary antibody conjugated to Alexa Fluor ${ }^{\circledR} 647$ (Invitrogen). Cells were then permeabilized with $0.5 \%$ saponin. After blocking with BSA, $\mathrm{Vpu}$, tetherin and TGN46 were stained with an anti-AU1 (Covance), anti-tetherin (eBioscience) and anti-TGN46 (Serotec) antibody, respectively. Secondary antibodies conjugated to Alexa Fluor ${ }^{\circledR}$ 568, Alexa Fluor ${ }^{\circledR} 647$ and Alexa Fluor $^{\circledR} 488$ were used for detection (Invitrogen). A confocal microscope (LSM 710, Zeiss) with the corresponding software (Zeiss Zen Software, 2010) was used for analysis.

\section{Click beetle assay}

The click beetle luciferase heteroprotein fragment complementation assay allows the real-time analysis of protein-protein interactions in living cells [33]. pCBGC_ß $3-T r C P 1$ and $\mathrm{p} ß$-catenin_CBG-N constructs encoding the $\mathrm{N}$ - or C-terminal fragment of click beetle green (CBG) were kindly provided by Piwnica-Worms. SalI and BamHI restriction sites were added to the vpu alleles by PCR and standard cloning techniques were used to insert the Vpu alleles into the $\mathrm{pCBG}-\mathrm{C}$ vector replacing the $B$-catenin gene. Splicing by overlap extension PCR (SOE-PCR) was used to fuse the C-terminal CBG-fragment to the C-terminus of human CD4. The B-TrCP1_CBG-C gene was replaced by the CD4_CBG-C fusion gene via HindIII and XbaI digestion. The luciferase assay was essentially performed as described before [33]. Briefly, 293T cells in white 96-well plates with clear bottom were transfected with equal amounts of the click beetle green constructs. 48 hours after transfection the cells were washed once and then incubated in MEBSS buffer (Modified Eagle's balanced salt solution: $144 \mathrm{mM}$ $\mathrm{NaCl}$, $5.4 \mathrm{mM} \mathrm{KCl}, 0.8 \mathrm{mM} \mathrm{MgSO}_{4}, 0.8 \mathrm{mM} \mathrm{NaH}_{2} \mathrm{PO}_{4}$, $1.2 \mathrm{mM} \mathrm{CaCl}_{2}$, glucose $5.6 \mathrm{mM}$, and HEPES $4 \mathrm{mM}$ [pH 7.4]) containing 1\% heat-inactivated fetal bovine serum and $150 \mu \mathrm{g} / \mathrm{ml}$ D-Luciferin. Photon flux was quantified at room temperature with a SAFAS Xenius spectrofluorimeter for $1 \mathrm{~min}$ or an Orion microplate luminometer for $10 \mathrm{sec}$.

\section{Sequence analysis}

$\mathrm{Vpu}$ amino acid sequences were aligned using multiple sequence alignment with hierarchical clustering (http:// multalin.toulouse.inra.fr/multalin/). Vpu sequences were obtained from the HIV Sequence Database (www.hiv.lanl.gov).

\section{Statistical methods}

The activities of vpu alleles were compared using a two-tailed Student's $t$ test. The PRISM package version 4.0 (Abacus Concepts, Berkeley, CA) was used for all calculations.

\section{Additional files}

\author{
Additional file 1: Table S1. Origin of HIV-1, SIVcpz and SIVgor vpu \\ alleles analyzed. \\ Additional file 2: Figure S1. Tetherin (upper panel) or CD4 \\ (lower panel) surface expression levels of HeLa cells co-transfected with a \\ CD4 (lower panel) expression vector and PCGCG plasmids expressing \\ eGFP alone (eGFPonly) or together with the indicated vpu alleles. A \\ construct expressing NL4-3 Vpu was used as a positive control.
}

Additional file 3: Figure S2. Levels of CD4 surface expression levels of 293T cells and HeLa cells transfected with a pCGCG plasmids expressing eGFP alone (vector) or together with CD4 and on TZM-bl cells stably expressing CD4. The numbers give the mean fluorescence intensity of CD4 expression by the transfected eGFP + cell population.

Additional file 4: Figure S3. Interaction of wild-type and chimeric HIV1 and SIVcpz/gor Vpu proteins with B-TrCP and CD4. Interaction of Vpu with $(A) B-\operatorname{Tr} C P$ and $(B, C) C D 4$ in the $(B)$ absence and $(C)$ presence of a dominant negative mutant of $\beta-\operatorname{TrCP} 1$. Refer to the legend to Figure 6 for further detail.

Competing interests

The authors declare that they have no competing interest.

\section{Authors' contributions}

SFK performed the experiments. MV, MP, YL, FBR and BHH contributed reagents. DS participated in the coordination of the study and in the review of the manuscript. FK designed and coordinated the study and wrote the final manuscript together with $\mathrm{BHH}$. All authors have read and approved the final manuscript.

\section{Acknowledgements}

We thank Susanne Engelhart and Martha Mayer for excellent technical assistance and Jan Münch and Christine Goffinet for critical reading of the manuscript. This work was supported by the Deutsche

Forschungsgemeinschaft (Leibniz award to FK) as well as grants from the $\mathrm{NIH}$ (R01 Al 50529, R01 Al 58715) and the Hector and Zeiss foundations.

\section{Author details}

${ }^{1}$ Institute of Molecular Virology, Ulm University Medical Center, 89081, Ulm, Germany. ${ }^{2} U M R$ 145, Institut de Recherche pour le Développement (IRD), Université de Montpellier, Montpellier, France. ${ }^{3}$ Departments of Medicine and Microbiology, University of Pennsylvania, Philadelphia, PA 19104, USA.

Received: 21 January 2013 Accepted: 8 March 2013

Published: 20 March 2013

\section{References}

1. Harris RS, Hultquist JF, Evans DT: The restriction factors of human immunodeficiency virus. J Biol Chem 2012, 287:40875-40883.

2. Blanco-Melo D, Venkatesh S, Bieniasz PD: Intrinsic cellular defenses against human immunodeficiency viruses. Immunity 2012, 37:399-411.

3. Malim MH, Bieniasz PD: HIV restriction factors and mechanisms of evasion. Cold Spring Harb Perspect Med 2012, 2:a006940. 
4. Kirchhoff F: Immune evasion and counteraction of restriction factors by HIV-1 and other primate lentiviruses. Cell Host Microbe 2010, 8:55-67.

5. Kratovac Z, Virgen CA, Bibollet-Ruche F, Hahn BH, Bieniasz PD, Hatziioannou T: Primate lentivirus capsid sensitivity to TRIM5 proteins. J Virol 2008, 82:6772-6777

6. Stremlau M, Owens CM, Perron MJ, Kiessling M, Autissier P, Sodroski J: The cytoplasmic body component TRIM5alpha restricts HIV-1 infection in Old World monkeys. Nature 2004, 427:848-853.

7. Roa A, Hayashi F, Yang Y, Lienlaf M, Zhou J, Shi J, Watanabe S, Kigawa T, Yokoyama S, Aiken C, Diaz-Griffero F: RING domain mutations uncouple TRIM5a restriction of HIV-1 from inhibition of reverse transcription and acceleration of uncoating. J Virol 2012, 86:1717-1727.

8. Gaddis NC, Sheehy AM, Ahmad KM, Swanson CM, Bishop KN, Beer BE, Marx PA, Gao F, Bibollet-Ruche F, Hahn BH, Malim MH: Further investigation of simian immunodeficiency virus Vif function in human cells. J Virol 2004, 78:12041-12046.

9. Sauter D, Schindler M, Specht A, Landford WN, Münch J, Kim K-A, Votteler J, Schubert U, Bibollet-Ruche F, Keele BF, Takehisa J, Ogando Y, Ochsenbauer C, Kappes JC, Ayouba A, Peeters M, Learn GH, Shaw G, Sharp PM, Bieniasz P, Hahn BH, Hatziioannou T, Kirchhoff F: Tetherin-driven adaptation of Vpu and Nef function and the evolution of pandemic and nonpandemic HIV-1 strains. Cell Host Microbe 2009, 6:409-421.

10. Jia B, Serra-Moreno R, Neidermyer W, Rahmberg A, Mackey J, Fofana IB, Johnson WE, Westmoreland S, Evans DT: Species-specific activity of SIV Nef and HIV-1 Vpu in overcoming restriction by tetherin/BST2. PLOS Pathog 2009, 5:e1000429.

11. Zhang F, Wilson SJ, Landford WC, Virgen B, Gregory D, Johnson MC, Munch J, Kirchhoff F, Bieniasz PD, Hatziioannou T: Nef proteins from simian immunodeficiency viruses are tetherin antagonists. Cell Host Microbe 2009, 6:54-67.

12. Sauter D, Specht A, Kirchhoff F: Tetherin: holding on and letting go. Cell 2010, 141:392-398

13. Sharp PM, Hahn BH: The evolution of HIV-1 and the origin of AIDS. Philos Trans R Soc Lond B Biol Sci 2010, 365:2487-2494.

14. Plantier J-C, Leoz M, Dickerson JE, De Oliveira F, Cordonnier F, Lemée V, Damond F, Robertson DL, Simon F: A new human immunodeficiency virus derived from gorillas. Nat Med 2009, 15:871-872.

15. Sauter D, Hué S, Petit SJ, Plantier J-C, Towers GJ, Kirchhoff F, Gupta RK: HIV-1 Group P is unable to antagonize human tetherin by Vpu. Env or Nef. Retrovirology 2011, 8:103.

16. Sauter D, Unterweger D, Vogl M, Usmani SM, Heigele A, Kluge SF, Hermkes E, Moll M, Barker E, Peeters M, Learn GH, Bibollet-Ruche F, Fritz JV, Fackler OT, Hahn BH, Kirchhoff F: Human tetherin exerts strong selection pressure on the HIV-1 group N Vpu protein. PLoS Pathog 2012, 8:e1003093.

17. Keele BF, Van Heuverswyn F, Li Y, Bailes E, Takehisa J, Santiago ML, BibolletRuche F, Chen Y, Wain LV, Liegeois F, Loul S, Ngole EM, Bienvenue Y, Delaporte E, Brookfield JFY, Sharp PM, Shaw GM, Peeters M, Hahn BH: Chimpanzee reservoirs of pandemic and nonpandemic HIV-1. Science 2006, 313:523-526.

18. Takehisa J, Kraus MH, Ayouba A, Bailes E, Van Heuverswyn F, Decker JM, Li Y, Rudicell RS, Learn GH, Neel C, Ngole EM, Shaw GM, Peeters M, Sharp PM Hahn $\mathrm{BH}$ : Origin and biology of simian immunodeficiency virus in wildliving western gorillas. J Virol 2009, 83:1635-1648.

19. Van Heuverswyn F, Li Y, Neel C, Bailes E, Keele BF, Liu W, Loul S, Butel C, Liegeois F, Bienvenue Y, Ngolle EM, Sharp PM, Shaw GM, Delaporte E, Hahn $\mathrm{BH}$, Peeters M: Human immunodeficiency viruses: SIV infection in wild gorillas. Nature 2006, 444:164.

20. Li Y, Ndjango J-B, Learn GH, Ramirez MA, Keele BF, Bibollet-Ruche F, Liu W, Easlick JL, Decker JM, Rudicell RS, Inogwabini B-I, Ahuka-Mundeke S, Leendertz FH, Reynolds V, Muller MN, Chancellor RL, Rundus AS, Simmons N, Worobey M, Shaw GM, Peeters M, Sharp PM, Hahn BH: Eastern chimpanzees, but not bonobos, represent a simian immunodeficiency virus reservoir. J Virol 2012, 86:10776-10791.

21. Kueck T, Neil SJD: A cytoplasmic tail determinant in HIV-1 Vpu mediates targeting of tetherin for endosomal degradation and counteracts interferon-induced restriction. PLoS Pathog 2012, 8:e1002609.

22. Petit SJ, Blondeau C, Towers GJ: Analysis of the human immunodeficiency virus type $1 \mathrm{M}$ group Vpu domains involved in antagonizing tetherin. J Gen Virol 2011, 92:2937-2948.

23. Ruiz A, Hill MS, Schmitt K, Guatelli J, Stephens EB: Requirements of the membrane proximal tyrosine and dileucine-based sorting signals for efficient transport of the subtype C Vpu protein to the plasma membrane and in virus release. Virology 2008, 378:58-68.

24. Dubé M, Roy BB, Guiot-Guillain P, Binette J, Mercier J, Chiasson A, Cohen EA: Antagonism of tetherin restriction of HIV-1 release by Vpu involves binding and sequestration of the restriction factor in a perinuclear compartment. PLoS Pathog 2010, 6:e1000856.

25. Sharp PM, Hahn BH: Origins of HIV and the AIDS pandemic. Cold Spring Harb Perspect Med 2011, 1:a006841.

26. Vigan R, Neil SJD: Separable determinants of subcellular localization and interaction account for the inability of group O HIV-1 Vpu to counteract tetherin. J Virol 2011, 85:9737-9748.

27. Andrew A, Strebel K: HIV-1 Vpu targets cell surface markers CD4 and BST-2 through distinct mechanisms. Mol Aspects Med 2010, 31:407-417.

28. Mitchell RS, Katsura C, Skasko MA, Fitzpatrick K, Lau D, Ruiz A, Stephens EB, Margottin-Goguet F, Benarous R, Guatelli JC: Vpu antagonizes BST-2 -mediated restriction of HIV-1 release via beta-TrCP and endo-lysosomal trafficking. PLoS Pathog 2009, 5:e1000450.

29. Kanemori Y, Uto K, Sagata N: Beta-TrCP recognizes a previously undescribed nonphosphorylated destruction motif in Cdc25A and Cdc25B phosphatases. Proc Natl Acad Sci U S A 2005, 102:6279-6284.

30. Bour S, Schubert U, Strebel $K$ : The human immunodeficiency virus type 1 Vpu protein specifically binds to the cytoplasmic domain of CD4: implications for the mechanism of degradation. J Virol 1995, 69:1510-1520.

31. Willey RL, Maldarelli F, Martin MA, Strebel K: Human immunodeficiency virus type 1 Vpu protein induces rapid degradation of CD4. J Virol 1992, 66:7193-7200

32. Margottin F, Bour SP, Durand H, Selig L, Benichou S, Richard V, Thomas D, Strebel K, Benarous R: A novel human WD protein, h-beta $\operatorname{TrCp}$, that interacts with HIV-1 Vpu connects CD4 to the ER degradation pathway through an F-box motif. Mol Cell 1998, 1:565-574.

33. Villalobos V, Naik S, Bruinsma M, Dothager RS, Pan M-H, Samrakandi M, Moss B, Elhammali A, Piwnica-Worms D: Dual-color click beetle luciferase heteroprotein fragment complementation assays. Chem Biol 2010, 17:1018-1029.

34. Douglas JL, Viswanathan K, McCarroll MN, Gustin JK, Früh K, Moses AV: Vpu directs the degradation of the human immunodeficiency virus restriction factor BST-2/Tetherin via a \{beta\}TrCP-dependent mechanism. J Virol 2009, 83:7931-7947.

35. Mangeat B, Gers-Huber G, Lehmann M, Zufferey M, Luban J, Piguet V: HIV-1 Vpu neutralizes the antiviral factor Tetherin/BST-2 by binding it and directing its beta-TrCP2-dependent degradation. PLOS Pathog 2009, 5:e1000574.

36. Kobayashi T, Ode H, Yoshida T, Sato K, Gee P, Yamamoto SP, Ebina H, Strebel $K$, Sato $H$, Koyanagi $Y$ : Identification of amino acids in the human tetherin transmembrane domain responsible for HIV-1 Vpu interaction and susceptibility. J Virol 2011, 85:932-945.

37. Schindler M, Münch J, Kutsch O, Li H, Santiago ML, Bibollet-Ruche F, MüllerTrutwin MC, Novembre FJ, Peeters M, Courgnaud V, Bailes E, Roques P Sodora DL, Silvestri G, Sharp PM, Hahn BH, Kirchhoff F: Nef-mediated suppression of $\mathrm{T}$ cell activation was lost in a lentiviral lineage that gave rise to HIV-1. Cell 2006, 125:1055-1067.

38. Bailes E, Gao F, Bibollet-Ruche F, Courgnaud V, Peeters M, Marx PA, Hahn $\mathrm{BH}$, Sharp PM: Hybrid origin of SIV in chimpanzees. Science 2003, 300:1713.

39. Moll M, Andersson SK, Smed-Sörensen A, Sandberg JK: Inhibition of lipid antigen presentation in dendritic cells by HIV-1 Vpu interference with CD1d recycling from endosomal compartments. Blood 2010, 116:1876-1884.

40. Shah AH, Sowrirajan B, Davis ZB, Ward JP, Campbell EM, Planelles V, Barker E: Degranulation of natural killer cells following interaction with HIV-1-infected cells is hindered by downmodulation of NTB-A by Vpu. Cell Host Microbe 2010, 8:397-409.

41. Doehle BP, Chang K, Rustagi A, McNevin J, McElrath MJ, Gale M Jr: Vpu mediates depletion of interferon regulatory factor 3 during HIV infection by a lysosome-dependent mechanism. J Virol 2012, 86:8367-8374.

42. Götz N, Sauter D, Usmani SM, Fritz JV, Goffinet C, Heigele A, Geyer M, Bibollet-Ruche F, Learn GH, Fackler OT, Hahn BH, Kirchhoff F: Reacquisition of Nef-mediated tetherin antagonism in a single in vivo passage of HIV-1 through its original chimpanzee host. Cell Host Microbe 2012, 12:373-380.

43. Kirchhoff F, Schindler M, Bailer N, Renkema GH, Saksela K, Knoop V, MüllerTrutwin MC, Santiago ML, Bibollet-Ruche F, Dittmar MT, Heeney JL, Hahn $\mathrm{BH}$, Münch J: Nef proteins from simian immunodeficiency virus-infected 
chimpanzees interact with p21-activated kinase 2 and modulate cell surface expression of various human receptors. J Virol 2004, 78:6864-6874.

44. Münch J, Schindler M, Wildum S, Rücker E, Bailer N, Knoop V, Novembre FJ, Kirchhoff F: Primary sooty mangabey simian immunodeficiency virus and human immunodeficiency virus type 2 nef alleles modulate cell surface expression of various human receptors and enhance viral infectivity and replication. J Virol 2005, 79:10547-10560.

45. Paul M, Jabbar MA: Phosphorylation of both phosphoacceptor sites in the HIV-1 Vpu cytoplasmic domain is essential for Vpu-mediated ER degradation of CD4. Virology 1997, 232:207-216.

46. Boll W, Rapoport I, Brunner C, Modis Y, Prehn S, Kirchhausen T: The mu2 subunit of the clathrin adaptor AP-2 binds to FDNPVY and Ypp $\varnothing$ sorting signals at distinct sites. Traffic 2002, 3:590-600.

47. Chen WJ, Goldstein JL, Brown MS: NPXY, a sequence often found in cytoplasmic tails, is required for coated pit-mediated internalization of the low density lipoprotein receptor. J Biol Chem 1990, 265:3116-3123.

48. Backer JM, Kahn CR, Cahill DA, Ullrich A, White MF: Receptor-mediated internalization of insulin requires a 12 -amino acid sequence in the juxtamembrane region of the insulin receptor beta-subunit. J Biol Chem 1990, 265:16450-16454.

49. Lim ES, Malik HS, Emerman M: Ancient adaptive evolution of tetherin shaped the functions of Vpu and Nef in human immunodeficiency virus and primate lentiviruses. $J$ Virol 2010, 84:7124-7134.

50. Gupta RK, Towers GJ: A tail of Tetherin: how pandemic HIV-1 conquered the world. Cell Host Microbe 2009, 6:393-395.

51. Papkalla A, Münch J, Otto C, Kirchhoff F: Nef enhances human immunodeficiency virus type 1 infectivity and replication independently of viral coreceptor tropism. J Virol 2002, 76:8455-8459.

52. Derdeyn CA, Decker JM, Sfakianos JN, Wu X, O'Brien WA, Ratner L, Kappes $J C$, Shaw GM, Hunter E: Sensitivity of human immunodeficiency virus type 1 to the fusion inhibitor T-20 is modulated by coreceptor specificity defined by the V3 loop of gp120. J Virol 2000, 74:8358-8367.

53. Platt EJ, Wehrly K, Kuhmann SE, Chesebro B, Kabat D: Effects of CCR5 and CD4 cell surface concentrations on infections by macrophagetropic isolates of human immunodeficiency virus type 1. J Virol 1998, 72:2855-2864.

54. Wei X, Decker JM, Liu H, Zhang Z, Arani RB, Kilby JM, Saag MS, Wu X, Shaw GM, Kappes JC: Emergence of resistant human immunodeficiency virus type 1 in patients receiving fusion inhibitor (T-20) monotherapy. Antimicrob Agents Chemother 2002, 46:1896-1905.

55. Münch J, Rajan D, Schindler M, Specht A, Rücker E, Novembre FJ, Nerrienet E, Müller-Trutwin MC, Peeters M, Hahn BH, Kirchhoff F: Nef-mediated enhancement of virion infectivity and stimulation of viral replication are fundamental properties of primate lentiviruses. J Virol 2007, 81:13852-13864.

56. Schindler M, Würfl S, Benaroch P, Greenough TC, Daniels R, Easterbrook P, Brenner M, Münch J, Kirchhoff F: Down-modulation of mature major histocompatibility complex class II and up-regulation of invariant chain cell surface expression are well-conserved functions of human and simian immunodeficiency virus nef alleles. J Virol 2003, 77:10548-10556.

doi:10.1186/1742-4690-10-32

Cite this article as: Kluge et al.: The transmembrane domain of HIV-1 Vpu is sufficient to confer anti-tetherin activity to SIVcpz and SIVgor Vpu proteins: cytoplasmic determinants of Vpu function. Retrovirology 2013 10:32.

\section{Submit your next manuscript to BioMed Central and take full advantage of:}

- Convenient online submission

- Thorough peer review

- No space constraints or color figure charges

- Immediate publication on acceptance

- Inclusion in PubMed, CAS, Scopus and Google Scholar

- Research which is freely available for redistribution

Submit your manuscript at www.biomedcentral.com/submit
C Biomed Central 